\title{
Impacts of the Higgs mass on vacuum stability, running fermion masses and two-body Higgs decays
}

\author{
Zhi-zhong Xing ${ }^{a *}$, He Zhang ${ }^{b \dagger}$, Shun Zhou ${ }^{c \ddagger}$ \\ ${ }^{a}$ Institute of High Energy Physics, Chinese Academy of Sciences, Beijing 100049, China \\ ${ }^{b}$ Max-Planck-Institut für Kernphysik, 69029 Heidelberg, Germany \\ ${ }^{c}$ Max-Planck-Institut für Physik (Werner-Heisenberg-Institut), 80805 München, Germany
}

\begin{abstract}
The recent results of the ATLAS and CMS experiments indicate $116 \mathrm{GeV} \lesssim$ $M_{H} \lesssim 131 \mathrm{GeV}$ and $115 \mathrm{GeV} \lesssim M_{H} \lesssim 127 \mathrm{GeV}$, respectively, for the mass of the Higgs boson in the standard model (SM) at the $95 \%$ confidence level. In particular, both experiments point to a preferred narrow mass range $M_{H} \simeq(124 \cdots 126) \mathrm{GeV}$. We examine the impact of this preliminary result of $M_{H}$ on the SM vacuum stability by using the two-loop renormalization-group equations (RGEs), and arrive at the cutoff scale $\Lambda_{\mathrm{VS}} \sim 4 \times 10^{12} \mathrm{GeV}$ (for $M_{H}=125 \mathrm{GeV}, M_{t}=172.9 \mathrm{GeV}$ and $\left.\alpha_{s}\left(M_{Z}\right)=0.1184\right)$ where the absolute stability of the SM vacuum is lost and some kind of new physics might take effect. We update the values of running lepton and quark masses at some typical energy scales, including the ones characterized by $M_{H}, 1 \mathrm{TeV}$ and $\Lambda_{\mathrm{VS}}$, with the help of the two-loop RGEs. The branching ratios of some important two-body Higgs decay modes, such as $H \rightarrow b \bar{b}, H \rightarrow \tau^{+} \tau^{-}, H \rightarrow \gamma \gamma$, $H \rightarrow W^{+} W^{-}$and $H \rightarrow Z Z$, are also recalculated by inputting the values of relevant particle masses at $M_{H}$.
\end{abstract}

PACS number(s): 12.15.Ff, 12.38.Bx, 14.80.Bn

Typeset using REVTEX

*E-mail: xingzz@ihep.ac.cn

†E-mail: he.zhang@mpi-hd.mpg.de

${ }^{\ddagger}$ E-mail: zhoush@mppmu.mpg.de 


\section{INTRODUCTION}

The Higgs mechanism [1] is responsible for the spontaneous $\mathrm{SU}(2)_{\mathrm{L}} \otimes \mathrm{U}(1)_{\mathrm{Y}} \rightarrow \mathrm{U}(1)_{\mathrm{em}}$ gauge symmetry breaking in the standard model (SM) of electroweak interactions [2], but the Higgs boson itself left no sort of trace in all the previous high-energy collider experiments. The main goal of the Large Hadron Collider (LHC) at CERN is just to discover this elusive particle, which allows other particles (except the photon and gluons) to gain finite masses. Combined with the indirect bounds obtained from the electroweak precision measurements, the recent data of the ATLAS and CMS experiments lead us to a rather narrow range of the Higgs mass: $114 \mathrm{GeV} \lesssim M_{H} \lesssim 141 \mathrm{GeV}$ [3]. In particular, both collaborations have reported their latest results

$$
M_{H} \simeq \begin{cases}(116 \cdots 131) \mathrm{GeV} & (\text { ATLAS [4]) } \\ (115 \cdots 127) \mathrm{GeV} & (\text { CMS [5]) }\end{cases}
$$

at the $95 \%$ confidence level. The ATLAS Collaboration has also found a preliminary hint of $M_{H} \simeq 126 \mathrm{GeV}$ with the $3.6 \sigma$ local significance in $H \rightarrow \gamma \gamma(2.8 \sigma), H \rightarrow Z Z^{*} \rightarrow 4 l$ $(2.1 \sigma)$ and $H \rightarrow W W^{*} \rightarrow 2 l 2 \nu(1.4 \sigma)$ decay modes [4]; and the CMS Collaboration has observed an excess compatible with $M_{H} \lesssim 124 \mathrm{GeV}$ with the $2.6 \sigma$ local significance [5]. These interesting results point to a preferred and narrower range $M_{H} \simeq(124 \cdots 126) \mathrm{GeV}$ for the SM Higgs boson. We are therefore confident that an unambiguous discovery of the Higgs boson will soon come true at the LHC.

Observing the Higgs boson and measuring its mass and other properties may help us solve several fundamental problems in elementary particle physics. Here we mention three of them for example.

- The Higgs mass theoretically suffers significant radiative corrections, and hence new symmetries and (or) new particles should be introduced to stabilize the electroweak scale $\Lambda_{\mathrm{EW}} \sim 10^{2} \mathrm{GeV}$ [6]. A solution to this gauge hierarchy problem calls for new physics beyond the SM, such as supersymmetries [7] or extra spatial dimensions [8].

- The Higgs boson is indispensable to the Yukawa interactions of three-family fermions which makes weak CP violation possible in the SM or its simple extensions [9]. To some extent, the existence of a Higgs boson may also support the Peccei-Quinn mechanism as an appealing solution to the strong CP problem [10].

- With the help of the Higgs field, one may write out the unique dimension-five operator $\ell \ell H H$ in an effective field theory [11] or implement the seesaw mechanism in a renormalizable quantum field theory [12] to generate finite but tiny neutrino masses.

Therefore, the highest priority of the LHC experiment is to pin down the Higgs boson and its quantum numbers. We are approaching a success in this connection.

Motivated by the encouraging ATLAS and CMS results, we aim to examine the impacts

of $M_{H} \simeq(124 \cdots 126) \mathrm{GeV}$ on the vacuum stability of the SM, the running behaviors of fermion masses and the branching ratios of the Higgs decays. The point is that a relatively small value of $M_{H}$ is likely to cause the vacuum instability unless new physics takes effect at a proper cutoff scale [13]. Given $M_{H} \simeq 125 \mathrm{GeV}$ as indicated by the latest LHC data, 
it is timely to determine the energy scale at which the effective quartic Higgs coupling $\tilde{\lambda}(\mu)$ runs to zero. We find that this cutoff scale is around $\Lambda_{\mathrm{VS}} \sim 4 \times 10^{12} \mathrm{GeV}$, which presumably signifies the end of the gauge desert and the beginning of a new physics oasis. Taking account of the allowed range of $M_{H}$ and the updated values of other SM parameters, we recalculate the running fermion masses at some typical energy scales up to $\Lambda_{\mathrm{VS}}$ by means of the renormalization-group equations (RGEs). Such an exercise makes sense because a sufficiently large value of $M_{H}$ (e.g., $M_{H} \simeq 140 \mathrm{GeV}$ ) was assumed in the previous works and hence the potential vacuum stability problem did not show up [14]. As a by-product, the branching ratios of some important two-body Higgs decay modes in the SM, such as $H \rightarrow b \bar{b}, H \rightarrow \tau^{+} \tau^{-}, H \rightarrow \gamma \gamma, H \rightarrow W^{+} W^{-}$and $H \rightarrow Z Z$, are also recalculated by using the new values of relevant particle masses obtained at $\mu \sim M_{H}$.

\section{THE HIGGS MASS AND VACUUM STABILITY}

First of all, let us briefly review the vacuum stability issue in the SM with a relatively light Higgs boson. In order to find out the true vacuum state and analyze its stability, one should calculate the effective scalar potential by taking account of radiative corrections and RGE improvements of the relevant parameters $[13,15]$. It has been shown that the $L$-loop scalar potential improved with $(L+1)$-loop RGEs actually includes all the $L$ th-to-leading logarithm contributions [16]. At the one-loop level, the effective scalar potential in the 't Hooft-Landau gauge and in the $\overline{\mathrm{MS}}$ renormalization scheme can be written as [17]

$$
\begin{aligned}
V_{\mathrm{eff}}[\phi(t)]= & -\frac{1}{2} m^{2}(t) \phi^{2}(t)+\frac{1}{4} \lambda(t) \phi^{4}(t)+\frac{3}{64 \pi^{2}}\left\{2 m_{W}^{4}[\phi(t)]\left[\ln \left(\frac{m_{W}^{2}[\phi(t)]}{\mu^{2}(t)}\right)-\frac{5}{6}\right]\right. \\
& \left.+m_{Z}^{4}[\phi(t)]\left[\ln \left(\frac{m_{Z}^{2}[\phi(t)]}{\mu^{2}(t)}\right)-\frac{5}{6}\right]-4 m_{t}^{4}[\phi(t)]\left[\ln \left(\frac{m_{t}^{2}[\phi(t)]}{\mu^{2}(t)}\right)-\frac{3}{2}\right]\right\},
\end{aligned}
$$

where the contributions from the Goldstone and Higgs bosons have been safely neglected, and $m_{W}^{2}[\phi(t)] \equiv g^{2}(t) \phi^{2}(t) / 4, m_{Z}^{2}[\phi(t)] \equiv\left[g^{2}(t)+g^{\prime 2}(t)\right] \phi^{2}(t) / 4$ and $m_{t}^{2}[\phi(t)] \equiv y_{t}^{2}(t) \phi^{2}(t) / 2$ have been defined. Note that the scale dependence of all the dimensionless couplings $\left(g(t), g^{\prime}(t), \lambda(t), y_{t}(t)\right)$, the mass parameter $m^{2}(t)$ and the Higgs field $\phi(t)$ has been explicitly indicated through the renormalization scale $\mu(t) \equiv M_{Z} e^{t}$ or equivalently the running parameter $t=\ln \left[\mu(t) / M_{Z}\right]$. The $\beta$ functions for the dimensionless couplings $\left(g(t), g^{\prime}(t), \lambda(t), y_{t}(t)\right)$ and the $\gamma$ functions for $\left(m^{2}(t), \phi(t)\right)$ at the two-loop order can be found in Refs. [17,18].

Due to the experimental observations, the scalar potential $V_{\text {eff }}$ must develop a realistic minimum at the electroweak scale, corresponding to the SM vacuum. Whether the SM vacuum is stable or not depends on the behavior of $V_{\text {eff }}$ in the large-field limit, i.e., $\phi(t) \gg$ $M_{Z}$. More explicitly, one can find out the extrema $\phi_{\mathrm{ex}}(t)$ of the scalar potential via

$$
\left.\frac{\partial V_{\mathrm{eff}}[\phi(t)]}{\partial \phi(t)}\right|_{\phi(t)=\phi_{\mathrm{ex}}(t)}=0 .
$$

At the weak scale $\mu\left(t_{Z}\right)=M_{Z}$, we should impose the boundary condition $\phi_{\text {ex }}\left(t_{Z}\right)=v \approx$ $246 \mathrm{GeV}$, which is the vacuum expectation value of the Higgs field. At the large-field values, the scalar potential is dominated by the quartic coupling term and the extrema $\phi_{\text {ex }}(t)$ can 
be evaluated at the renormalization scale $\mu(t)=\phi_{\mathrm{ex}}(t)$ from Eqs. (2) and (3) as $\phi_{\mathrm{ex}}^{2}=m^{2} / \tilde{\lambda}$, where the effective quartic coupling $\tilde{\lambda}$ is defined as

$$
\begin{aligned}
\tilde{\lambda}=\lambda & -\frac{3}{32 \pi^{2}}\left\{\frac{1}{8}\left(g^{\prime 2}+g^{2}\right)^{2}\left[\frac{1}{3}-\ln \left(\frac{g^{2}+g^{2}}{4}\right)\right]\right. \\
& \left.+2 y_{t}^{4}\left[\ln \left(\frac{y_{t}^{2}}{2}\right)-1\right]+\frac{1}{4} g^{4}\left[\frac{1}{3}-\ln \left(\frac{g^{2}}{4}\right)\right]\right\} .
\end{aligned}
$$

Now it is clear that $V_{\text {eff }} \approx \tilde{\lambda} \phi^{4} / 4$ will develop a minimum much deeper than the realistic minimum if the effective coupling $\tilde{\lambda}$ becomes negative [19-23]. To maintain the absolute stability of the SM vacuum, new physics should come into play below or at the energy scale $\Lambda_{\mathrm{VS}}$ where the effective coupling $\tilde{\lambda}$ vanishes, i.e., $\tilde{\lambda}\left(\Lambda_{\mathrm{VS}}\right)=0$. One can derive a lower mass bound on the Higgs boson by requiring that the SM vacuum is absolutely stable up to a possible grand-unified-theory (GUT) scale or the Planck scale [13,19-23].

In view of the allowed range of the Higgs mass, we may conversely implement the vacuum stability argument to determine the energy scale $\Lambda_{\mathrm{VS}}$ at which new physics should take effect. Our strategy is as follows. First, we have to specify the matching conditions relating the quartic coupling $\lambda$ to the Higgs mass $M_{H}$, as well as the top-quark Yukawa coupling $y_{t}$ to the top-quark pole mass $M_{t}$. Although the complete effective potential $V_{\text {eff }}$ must be scaleindependent, the one with one-loop approximation is not. The solution is to find an optimal scale $\mu^{*}=\mu\left(t^{*}\right)$ for which the effective potential has the least scale-dependence, as shown in Ref. [19], where one can observe that $\mu^{*}=M_{t}$ is a reasonable choice. Therefore, we choose the matching conditions for $\lambda$ and $y_{t}$ at $\mu^{*}=M_{t}$ :

$$
\begin{aligned}
\lambda\left(M_{t}\right) & =\frac{M_{H}^{2}}{2 v^{2}}\left[1+\delta_{H}\left(M_{t}\right)\right], \\
y_{t}\left(M_{t}\right) & =\frac{\sqrt{2} M_{t}}{v}\left[1+\delta_{t}\left(M_{t}\right)\right],
\end{aligned}
$$

where the correction terms $\delta_{H}\left(M_{t}\right)$ and $\delta_{t}\left(M_{t}\right)$ have been given in Ref. [23-25]. The values of the other input parameters are taken from Ref. [26] and will be specified in Sec. III when we turn to the running fermion masses. Second, we run $\lambda(\mu)$ to a much higher energy scale by solving the complete two-loop RGEs. Third, the cutoff scale $\Lambda_{\mathrm{VS}}$ can be identified with the solution to $\tilde{\lambda}\left(\Lambda_{\mathrm{VS}}\right)=0$, where $\tilde{\lambda}$ and $\lambda$ are related via Eq. (4). Note that the cutoff scale $\Lambda_{\mathrm{VS}}$ determined by $\tilde{\lambda}\left(\Lambda_{\mathrm{VS}}\right)=0$ could be an order of magnitude larger than the one by $\lambda\left(\Lambda_{\mathrm{VS}}\right)=0$, which has not taken account of the one-loop radiative corrections to the scalar potential $[19,21]$.

Our numerical result for the correlation between the Higgs mass and the energy scale is shown in FIG. 1. Some comments are in order.

1. If $M_{H} \gtrsim 129 \mathrm{GeV}$ holds, the vacuum stability can be guaranteed even around a possible GUT scale (e.g., $10^{16} \mathrm{GeV}$ ) or the Planck scale $\Lambda_{\mathrm{Pl}} \sim 10^{19} \mathrm{GeV}$ [27]. The cutoff scale $\Lambda_{\mathrm{VS}}$ increases as the Higgs mass $M_{H}$ increases, but this observation is sensitively dependent on the value of the top-quark pole mass $M_{t}$. 
2. Given $M_{H} \simeq 125 \mathrm{GeV}$, some kind of new physics should come out around $\Lambda_{\mathrm{VS}} \sim$ $10^{12} \mathrm{GeV}$ to stabilize the SM vacuum ${ }^{1}$. For example, it is interesting to notice that the canonical seesaw mechanism for neutrino mass generation is expected to work around this cutoff scale. In such a seesaw model the heavy Majorana neutrinos could have masses of $\mathcal{O}\left(10^{12}\right) \mathrm{GeV}$, so that the leptogenesis mechanism [28] may work well to account for the observed matter-antimatter asymmetry of the Universe.

Although the existence of a cutoff scale is robust for the SM with a relatively light Higgs boson, it remains unclear what kind of new physics could take effect over there. In any event, if the new physics responsible for the vacuum stability could also offer a solution to the flavor puzzles of leptons and quarks (especially the origin of tiny neutrino masses), the running fermion masses at the cutoff scale $\Lambda_{\mathrm{VS}}$ will be very helpful for model building. We shall focus on this issue in the following section.

\section{RUNNING LEPTON AND QUARK MASSES}

A systematic analysis of the RGE running masses of leptons and quarks has been done in Ref. [14], where $M_{H} \simeq 140 \mathrm{GeV}$ has typically been taken just for illustration. As discussed above, such a value of the Higgs mass makes the situation simple because it does not give rise to the vacuum instability problem in the SM. Here we want to update the running fermion masses for two good reasons: (a) the latest ATLAS and CMS data point to $M_{H} \simeq$ $(124 \cdots 126) \mathrm{GeV}$, and hence the issue of vacuum stability should be taken seriously; (b) the values of some of the input parameters adopted in Ref. [14] have more or less changed in the past few years, and thus an update of the analysis is necessary. Before doing a detailed RGE analysis of fermion masses, let us summarize the input parameters and outline our calculational strategy.

- Six quark masses are $m_{u}(2 \mathrm{GeV})=(1.7 \cdots 3.1) \mathrm{MeV}, m_{d}(2 \mathrm{GeV})=(4.1 \cdots 5.7) \mathrm{MeV}$, $m_{s}(2 \mathrm{GeV})=(80 \cdots 130) \mathrm{MeV}, m_{c}\left(m_{c}\right)=1.29_{-0.11}^{+0.05} \mathrm{GeV}, m_{b}\left(m_{b}\right)=4.19_{-0.16}^{+0.08} \mathrm{GeV}$ and $M_{t}=172.9_{-1.1}^{+1.1} \mathrm{GeV}[26]$, where $M_{t}$ represents the pole mass of the top quark extracted from the direct measurements. In addition, the pole masses of three charged leptons are given by $M_{e}=(0.510998910 \pm 0.000000013) \mathrm{MeV}, M_{\mu}=(105.658367 \pm 0.000004) \mathrm{MeV}$ and $M_{\tau}=(1776.82 \pm 0.16) \mathrm{MeV}[26]$. Following the same approach as the one described in Ref. [14], we can calculate the running masses of charged leptons and quarks at some typical energy scales in the $\mathrm{SM}$, including $\mu=M_{W}, M_{Z}, M_{H}, 1 \mathrm{TeV}$ and $\Lambda_{\mathrm{VS}}$.

- The strong and electromagnetic fine-structure constants at $M_{Z}$ are $\alpha_{s}\left(M_{Z}\right)=0.1184 \pm$ 0.0007 and $\alpha\left(M_{Z}\right)^{-1}=127.916 \pm 0.015$, and the weak mixing angle is $\sin ^{2} \theta_{W}\left(M_{Z}\right)=$

\footnotetext{
${ }^{1}$ Since the cutoff scale depends sensitively on the Higgs mass in the range $[120 \mathrm{GeV}, 130 \mathrm{GeV}]$, as shown in FIG. 1, one has to take care of experimental errors from the top quark mass $M_{t}$ and the strong coupling $\alpha_{s}\left(M_{Z}\right)$, as well as the theoretical uncertainties involved in the two-loop RGEs and one-loop matching conditions [21]. For instance, the $\mathrm{SM}$ vacuum for $M_{H} \simeq 125 \mathrm{GeV}$ could even be stable up to the Planck scale $\Lambda_{\mathrm{Pl}} \simeq 10^{19} \mathrm{GeV}$ if the relevant uncertainties are included.
} 
$0.23116 \pm 0.00013[26]$. With the help of these input parameters, one may determine the gauge coupling constants $g_{s}^{2}=4 \pi \alpha_{s}, g^{2}=4 \pi \alpha / \sin ^{2} \theta_{W}$ and $g^{\prime}=g \tan \theta_{W}$ at the energy scale $\mu=M_{Z}$.

- The four parameters of quark flavor mixing and $\mathrm{CP}$ violation in the modified Wolfenstein parametrization are $\lambda=0.2253 \pm 0.0007, A=0.808_{-0.015}^{+0.022}, \bar{\rho}=0.132_{-0.014}^{+0.022}$ and $\bar{\eta}=0.341 \pm 0.013$ [26]. These values, together with the values of quark masses, allow us to reconstruct the quark Yukawa coupling matrices $Y_{\mathrm{u}}$ and $Y_{\mathrm{d}}$ at the electroweak scale. The RGEs of $Y_{\mathrm{u}}$ and $Y_{\mathrm{d}}$ can therefore help us to run the quark masses and flavor mixing parameters to a much higher energy scale.

- The allowed ranges of three lepton flavor mixing angles are $30.6^{\circ} \leq \theta_{12} \leq 36.8^{\circ}$, $35.7^{\circ} \leq \theta_{23} \leq 53.1^{\circ}$ and $1.8^{\circ} \leq \theta_{13} \leq 12.1^{\circ}[29]$, and the allowed ranges of two neutrino mass-squared differences are $6.99 \times 10^{-5} \mathrm{eV}^{2} \leq \delta m^{2} \leq 8.18 \times 10^{-5} \mathrm{eV}^{2}$ and $2.06 \times 10^{-3} \mathrm{eV}^{2} \leq\left|\Delta m^{2}\right| \leq 2.67 \times 10^{-3} \mathrm{eV}^{2}[29]$. For simplicity, we only take the bestfit values $\theta_{12}=33.6^{\circ}, \theta_{23}=40.4^{\circ}$ and $\theta_{13}=8.3^{\circ}$ together with $\delta m^{2}=7.58 \times 10^{-5} \mathrm{eV}^{2}$ and $\left|\Delta m^{2}\right|=2.35 \times 10^{-3} \mathrm{eV}^{2}$ as the inputs at $M_{Z}$ in our numerical calculations. In particular, the value of $\theta_{13}$ taken above is essentially consistent with the latest Daya Bay [30] and RENO [31] results. The unknown CP-violating phases in the lepton sector are all assumed to be zero. In view of the fact that the absolute neutrino mass scale is also unknown, we shall only consider the normal mass hierarchy with $m_{1}=0.001 \mathrm{eV}$ and $m_{1}<m_{2} \ll m_{3}$ at $M_{Z}$ for illustration. For the same reason, only the one-loop $\mathrm{RGE}$ for neutrino masses is considered. It is then possible to reconstruct the chargedlepton Yukawa coupling matrix $Y_{l}$ and the effective neutrino coupling matrix $\kappa$ at $M_{Z}$ from the given lepton masses and flavor mixing parameters [32].

For a complete list of the RGEs to be used in our numerical analysis, we refer the reader to Ref. [14] and references therein.

TABLES I and II summarize our numerical results for the running quark and chargedlepton masses at some typical energy scales, respectively. Different from the previous works, here the scales characterized by the Higgs mass $M_{H}$ and the vacuum stability cutoff $\Lambda_{\mathrm{VS}}$ are taken into account for the first time. The values of fermion masses at $M_{H}$ will be used to calculate the branching ratios of some important Higgs decay modes in Sec. IV, and those at $\Lambda_{\mathrm{VS}}$ are expected to be very useful for building possible flavor models beyond the SM.

In studying the running behaviors of twelve fermion masses above $M_{Z}$, we have used the inputs at $M_{Z}$ to numerically solve the RGEs of the Yukawa coupling matrices $Y_{\mathrm{u}}, Y_{\mathrm{d}}, Y_{l}$ and the effective neutrino coupling matrix $\kappa$ as well as the two-loop RGEs of the quartic Higgs coupling $\lambda(\mu)$ and gauge couplings at $\mu \geq M_{Z}$. After $Y_{\mathrm{u}}, Y_{\mathrm{d}}, Y_{l}$ and $\kappa$ are diagonalized, one can obtain the running quark masses $m_{q}(\mu)=y_{q}(\mu) v / \sqrt{2}$ (for $q=u, c, t$ and $d, s, b$ ), the running charged-lepton masses $m_{l}(\mu)=y_{l}(\mu) v / \sqrt{2}$ (for $l=e, \mu, \tau$ ) and the running neutrino masses $m_{i}(\mu)=\kappa_{i}(\mu) v^{2} / 2$ (for $i=1,2,3$ ). The corresponding quark and lepton flavor mixing parameters can simultaneously be achieved. For simplicity, let us define $R_{f}(\mu) \equiv$ $m_{f}(\mu) / m_{f}\left(M_{Z}\right)$, where the subscript $f$ runs over the mass-eigenstate indices of six quarks and six leptons. We find that $R_{u}(\mu) \approx R_{d}(\mu) \approx R_{s}(\mu) \approx R_{c}(\mu) \approx R_{e}(\mu) \approx R_{\mu}(\mu) \approx 1$ holds to a good degree of accuracy, if $\mu$ is below the cutoff scale $\Lambda_{\mathrm{VS}}$. So we only plot the 
numerical results of $R_{t}(\mu), R_{b}(\mu)$ and $R_{\tau}(\mu)$ in FIG. 2. The ratios $R_{i}(\mu)$ for three neutrino masses are shown in FIG. 3. Some comments are in order.

1. The mass ratios $R_{f}(\mu)$ are not very sensitive to the quartic Higgs coupling $\lambda(\mu)$ or equivalently the Higgs mass $M_{H}$, simply because the latter enters the RGEs of fermion masses only at the two-loop level. As observed in Ref. [14], there exists a maximum for the charged-lepton masses around $\mu \sim 10^{6} \mathrm{GeV}$, while the quark masses monotonously decrease as the energy scale increases. Taking account of the vacuum instability problem discussed in Sec. II, we argue that the evolution of fermion masses above the cutoff scale $\Lambda_{\mathrm{VS}}$ might not be meaningful anymore. We expect that some kind of new physics should take effect around $\Lambda_{\mathrm{VS}}$ and thus modify the RGEs of the SM.

2. In most cases the running behaviors of three neutrino masses are neither sensitive to their absolute values nor sensitive to their mass hierarchies in the SM [33]. Only when three neutrino masses are assumed to be nearly degenerate, the RGE running effects of neutrino mass and mixing parameters are possible to be significant. But the dependence of $m_{i}(\mu)$ on the quartic Higgs coupling $\lambda(\mu)$ or the Higgs mass $M_{H}$ is quite evident, simply because the effective neutrino coupling matrix $\kappa$ receives the one-loop corrections from the quartic Higgs interaction [14,33].

For simplicity, we skip the numerical illustration of the running behaviors of quark and lepton flavor mixing parameters in this paper.

\section{BRANCHING RATIOS OF THE HIGGS DECAYS}

The present ATLAS and CMS experiments are mainly sensitive to the Higgs boson via its decay channels $H \rightarrow \gamma \gamma, H \rightarrow b \bar{b}, H \rightarrow \tau^{+} \tau^{-}, H \rightarrow W^{+} W^{-}(2 l 2 \nu)$ and $H \rightarrow$ $Z Z(4 l, 2 l 2 \nu, 2 l 2 q, 2 l 2 \tau)$, where $l=e$ or $\mu$ and $\nu$ denotes the neutrinos of any flavors [3]. Which channel is dominant depends crucially on the Higgs mass. If $M_{H} \lesssim 135 \mathrm{GeV}$ holds, the decay mode $H \rightarrow b \bar{b}$ is expected to have the largest branching ratio; and if the Higgs mass is slightly heavier, the decay mode $H \rightarrow W^{+} W^{-}$will surpass the others [15].

We first consider the leptonic $H \rightarrow l^{+} l^{-}$decays, where $l$ runs over $e, \mu$ or $\tau$. Including the one-loop electroweak corrections, the decay width of $H \rightarrow l^{+} l^{-}$is given by [34]

$$
\Gamma_{l}=\frac{G_{\mathrm{F}} M_{H}}{4 \sqrt{2} \pi} M_{l}^{2}\left(1-\frac{4 M_{l}^{2}}{M_{H}^{2}}\right)^{3 / 2}\left(1+\delta_{\mathrm{QED}}+\delta_{\mathrm{W}}\right)
$$

where $G_{\mathrm{F}}$ is the Fermi constant, $\delta_{\mathrm{QED}}=9 \alpha\left[3-2 \ln \left(M_{H}^{2} / M_{l}^{2}\right)\right] /(12 \pi)$, and

$$
\delta_{\mathrm{W}}=\frac{G_{\mathrm{F}}}{8 \sqrt{2} \pi^{2}}\left\{7 M_{t}^{2}+M_{W}^{2}\left(\frac{3}{\sin ^{2} \theta_{W}} \ln \cos ^{2} \theta_{W}-5\right)-M_{Z}^{2}\left[3\left(1-4 \sin ^{2} \theta_{W}\right)^{2}-\frac{1}{2}\right]\right\} .
$$

Note that the large logarithmic term $\ln \left(M_{H}^{2} / M_{l}^{2}\right)$ in $\delta_{\text {QED }}$ can be absorbed in the running mass of $l$ at the scale of $M_{H}$, which has been given in TABLE II.

Now we turn to the $H \rightarrow q \bar{q}$ decays, where $q$ runs over $u, d, s, c$ or $b$ for the Higgs mass to lie in the range $114 \mathrm{GeV} \lesssim M_{H} \lesssim 141 \mathrm{GeV}$. Since the decay rates of $H \rightarrow u \bar{u}, d \bar{d}$ and $s \bar{s}$ 
are highly suppressed by the corresponding quark masses, we are mainly interested in the decay rates of $H \rightarrow c \bar{c}$ and $H \rightarrow b \bar{b}$. Up to the three-loop QCD corrections [35],

$$
\Gamma_{q}=\frac{3 G_{\mathrm{F}} M_{H}}{4 \sqrt{2} \pi} m_{q}^{2}\left(M_{H}\right)\left(\delta_{\mathrm{QCD}}+\delta_{t}\right)
$$

where

$$
\delta_{\mathrm{QCD}}=1+5.67\left(\frac{\alpha_{s}\left(M_{H}\right)}{\pi}\right)+29.14\left(\frac{\alpha_{s}\left(M_{H}\right)}{\pi}\right)^{2}+41.77\left(\frac{\alpha_{s}\left(M_{H}\right)}{\pi}\right)^{3}
$$

and

$$
\delta_{t}=\left(\frac{\alpha_{s}\left(M_{H}\right)}{\pi}\right)^{2}\left[1.57-\frac{2}{3} \ln \left(\frac{M_{H}^{2}}{M_{t}^{2}}\right)+\frac{1}{9} \ln ^{2}\left(\frac{m_{q}^{2}\left(M_{H}\right)}{M_{H}^{2}}\right)\right] .
$$

Note that the running quark masses $m_{q}\left(M_{H}\right)$ and the strong coupling constant $\alpha_{s}\left(M_{H}\right)$ at the scale of $M_{H}$ are useful here to absorb the large logarithmic terms.

A detailed discussion about the two-body decay modes $H \rightarrow \gamma \gamma, H \rightarrow W^{+} W^{-}, H \rightarrow$ $Z Z, H \rightarrow g g$ and $H \rightarrow t \bar{t}$ can be found in Ref. [36]. For simplicity, here we do not elaborate the relevant analytical results but do a numerical recalculation based on the updated particle masses at $M_{H}$. In order to compute the branching ratios of the above decay channels, we implement the latest version of the program HDECAY [37] and update the input parameters according to our TABLES I and II together with Ref. [26]. Some comments are in order.

- The pole masses of the charged leptons (i.e., $M_{l}$ ), instead of the running masses $m_{l}\left(M_{H}\right)$, have been used as the input parameters in the program HDECAY. This treatment is consistent with the formula of $\Gamma_{l}$ given in Eq. (6). If one chooses to use the running masses $m_{l}\left(M_{H}\right)$ in the numerical calculation, then the correction terms in Eq. (6) should take different forms.

- The one-loop pole masses of $c$ and $b$ quarks have been used as the input parameters in the program HDECAY, because they must be consistent with the corresponding parton distribution function when the production of the Higgs boson in a hadron collider (e.g., the LHC) is taken into account [38]. In our calculations we start from the values of $m_{c}\left(m_{c}\right)$ and $m_{b}\left(m_{b}\right)[26]$ and then evaluate the pole masses $M_{c}$ and $M_{b}$ as precisely as possible by using the relevant four-loop RGEs and three-loop matching conditions [14]. Hence we obtain the pole masses $M_{c}=1.84 \mathrm{GeV}$ and $M_{b}=4.92 \mathrm{GeV}$, as given in TABLE I.

Our numerical results for the branching ratios of $H \rightarrow b \bar{b}, c \bar{c}$ and $\tau^{+} \tau^{-}$decays are shown in FIG. 4, where the branching ratios of $H \rightarrow \gamma \gamma, g g, W^{+} W^{-}, Z Z$ and $Z \gamma$ decays are also plotted for a comparison. These important two-body decay channels will help discover the Higgs boson and pin down its mass in the near future. The branching ratios of $H \rightarrow s \bar{s}$ and $H \rightarrow \mu^{+} \mu^{-}$are of $\mathcal{O}\left(10^{-4}\right)$ in the range of $M_{H} \in[110 \mathrm{GeV}, 150 \mathrm{GeV}]$, and thus they have been neglected from FIG. 4. 


\section{SUMMARY}

In view of the recent results from the ATLAS and CMS experiments which hint at the existence of the Higgs boson, we have examined the impact of the Higg mass on vacuum stability in the SM by means of the two-loop RGEs. We find that $M_{H} \simeq 125 \mathrm{GeV}$ leads us to an interesting cutoff scale $\Lambda_{\mathrm{VS}} \sim 10^{12} \mathrm{GeV}$, as required by the vacuum stability. Some kind of new physics are therefore expected to take effect around $\Lambda_{\mathrm{VS}}$. In other words, $\Lambda_{\mathrm{VS}}$ characterizes the end of the gauge desert and the beginning of a new physics oasis.

We have argued that possible new physics responsible for the vacuum stability of the SM might also be able to help solve the flavor puzzles. Hence we have recalculated the running fermion masses up to the cutoff scale $\Lambda_{\mathrm{VS}}$ by inputting the allowed range of $M_{H}$ and the updated values of other SM parameters into the full set of the two-loop RGEs for the quartic Higgs coupling, the Yukawa couplings and the gauge couplings. In particular, the values of lepton and quark masses at $\mu=M_{H}$ and $\Lambda_{\mathrm{VS}}$ are obtained for the first time. As a by-product, the branching ratios of some important two-body Higgs decay modes in the SM, such as $H \rightarrow b \bar{b}, H \rightarrow \tau^{+} \tau^{-}, H \rightarrow \gamma \gamma, H \rightarrow W^{+} W^{-}$and $H \rightarrow Z Z$, have been recalculated with the help of the new values of relevant particle masses obtained at $M_{H}$. Our numerical results should be very useful for model building and flavor physics.

We reiterate that an unambiguous discovery of the Higgs boson at the LHC in the near future will pave the way for us to confirm the Yukawa interactions between the Higgs field and fermion fields. That will be a crucial step towards understanding the origin of fermion masses, flavor mixing and CP violation either within or beyond the SM. This point is especially true for testing the seesaw mechanisms, which attribute the tiny masses of three known neutrinos to the presence of some unknown heavy degrees of freedom via the Yukawa interactions. We believe that a new era of flavor physics is coming to the surface.

The authors are indebted to Peter Zerwas for valuable comments and suggestions. This work was supported in part by the National Natural Science Foundation of China under grant No. 11135009 and by the Ministry of Science and Technology of China under grant No. 2009CB825207 (Z.Z.X.), by the ERC under the Starting Grant MANITOP and by the DFG in the Transregio 27 "Neutrinos and Beyond" (H.Z.) and by the Alexander von Humboldt Foundation (S.Z.). 


\section{REFERENCES}

[1] P. Higgs, Phys. Lett. B 12, 132 (1964); Phys. Rev. Lett. 13, 508 (1964); F. Englert and R. Brout, Phys. Rev. Lett. 13, 321 (1964); G.S. Guralnik, C.R. Hagen, and T.W.B. Kibble, Phys. Rev. Lett. 13, 585 (1964); P. Higgs, Phys. Rev. 145, 1156 (1966).

[2] S.L. Glashow, Nucl. Phys. 22, 579 (1961); S. Weinberg, Phys. Rev. Lett. 19, 1264 (1967); A. Salam, in Elementary Particle Theory, edited by N. Svartholm (Almqvist and Wiksells, Stockholm, 1968), p. 367.

[3] The ATLAS and CMS Collaborations, Combined Standard Model Higgs boson searches with up to $2.3 \mathrm{fb}^{-1}$ of pp collision data at $\sqrt{s}=7$ TeV at the LHC, ATLAS-CONF2011-157, CMS PAS HIG-11-023 (2011).

[4] G. Aad et al. (the ATLAS Collaboration), Phys. Lett. B 710, 49 (2012).

[5] S. Chatrchyan et al. (the CMS Collaboration), Phys. Lett. B 710, 26 (2012).

[6] S. Weinberg, Phys. Rev. D 13, 974 (1976); Phys. Rev. D 19, 1277 (1979); E. Gildener, Phys. Rev. D 14, 1667 (1976); L. Susskind, Phys. Rev. D 20, 2619 (1979); G. 't Hooft, in Recent developments in gauge theories, Proceedings of the NATO Advanced Summer Institute, Cargese 1979, (Plenum, 1980).

[7] H.P. Nilles, Phys. Rept. 110, 1 (1984); H.E. Haber and G.L. Kane, Phys. Rept. 117, 75 (1985); M.F. Sohnius, Phys. Rept. 128, 39 (1985).

[8] N. Arkani-Hamed, S. Dimopoulos, and G. Dvali, Phys. Lett. B 429, 263 (1998); Phys. Rev. D 59, 086004 (1999).

[9] M. Kobayashi and T. Maskawa, Prog. Theor. Phys. 49, 652 (1973).

[10] R.D. Peccei and H.R. Quinn, Phys. Rev. Lett. 38, 1440 (1977).

[11] S. Weinberg, Phys. Rev. Lett. 43, 1566 (1979).

[12] P. Minkowski, Phys. Lett. B 67, 421 (1977); T. Yanagida, in Proceedings of the Workshop on Unified Theory and the Baryon Number of the Universe, edited by O. Sawada and A. Sugamoto (KEK, Tsukuba, 1979); M. Gell-Mann, P. Ramond, and R. Slansky, in Supergravity, edited by P. van Nieuwenhuizen and D. Freedman (North Holland, Amsterdam, 1979); S.L. Glashow, in Quarks and Leptons, edited by M. Lévy et al. (Plenum, New York, 1980); R.N. Mohapatra and G. Senjanovic, Phys. Rev. Lett. 44, 912 (1980).

[13] A.D. Linde, JETP Lett. 23, 64 (1976); Phys. Lett. B 62, 435 (1976); S. Weinberg, Phys. Rev. Lett. 36, 294 (1976); H.D. Politzer and S. Wolfram, Phys. Lett. B 82, 242 (1979); P.Q. Hung, Phys. Rev. Lett. 42, 873 (1979); N. Cabibbo et al., Nucl. Phys. B 158, 295 (1979); R.A. Flores and M. Sher, Phys. Rev. D 27, 1679 (1983). For a review, see: M. Sher, Phys. Rept. 179, 273 (1989).

[14] Z.Z. Xing, H. Zhang, and S. Zhou, Phys. Rev. D 77, 113016 (2008).

[15] J.F. Gunion et al., The Higgs Hunter's Guide (Perseus Publishing, Cambridge, Massachusetts, 1990); A. Djouadi, Phys. Rept. 457, 1 (2008).

[16] M. Bando et al., Phys. Lett. B 301, 83 (1993); Prog. Theor. Phys. 90, 405 (1993).

[17] C. Ford et al., Nucl. Phys. B 395, 17 (1993).

[18] M.E. Machacek and M.T. Vaughn, Nucl. Phys. B 222, 83 (1984); 236, 221 (1984); 249, 70 (1985); H. Arason et al., Phys. Rev. D 46, 3945; M.X. Luo and Y. Xiao, Phys. Rev. Lett. 90, 011601 (2003).

[19] J.A. Casas, J.R. Espinosa, and M. Quiros, Phys. Lett. B 342, 171 (1995).

[20] G. Altarelli and G. Isidori, Phys. Lett. B 337, 141 (1994). 
[21] J.A. Casas, J.R. Espinosa, and M. Quiros, Phys. Lett. B 382, 374 (1996); G. Isidori, G. Ridolfi, and A. Strumia, Nucl. Phys. B 609, 387 (2001); G. Isidori et al., Phys. Rev. D 77, 025034 (2008); J. Ellis et al., Phys. Lett. B 679, 369 (2009); J. Elias-Miro et al., Phys. Lett. B 709, 222 (2012).

[22] L. Maiani, G. Parisi, and R. Petronzio, Nucl. Phys. B 136, 115 (1979); R. Dashen and H. Neuberger, Phys. Rev. Lett. 50, 1897 (1983); D.J.E. Callaway, Nucl. Phys. B 233, 189 (1984); M.A. Beg, C. Panagiotakopolous, and A. Sirlin, Phys. Rev. Lett. 52, 883 (1984); M. Lindner, Z. Phys. C 31, 295 (1986).

[23] T. Hambye and K. Riesselmann, Phys. Rev. D 55, 7255 (1996).

[24] A. Sirlin and R. Zucchini, Nucl. Phys. B 266, 389 (1986).

[25] R. Hempfling, and B.A. Kniehl, Phys. Rev. D 51, 1386 (1995).

[26] K. Nakamura et al., J. Phys. G 37, 075021 (2010); and update for the 2012 edition.

[27] See, e.g., M. Holthausen, K.S. Lim, and M. Lindner, JHEP 1202, 037 (2012); I. Masina and A. Notari, arXiv:1112.2659.

[28] M. Fukugita and T. Yanagida, Phys. Lett. B 174, 45 (1986).

[29] G.L. Fogli et al., Phys. Rev. D 84, 053007 (2011); See also T. Schwetz, M. Tortola, and J.W.F. Valle, New J. Phys 13, 109401 (2011).

[30] F.P. An et al. (Daya Bay Collaboration), arXiv:1203.1669 (Phys. Rev. Lett. in press).

[31] J.K. Ahn et al. (RENO Collaboration), arXiv:1204.0626.

[32] Here we simply assume that finite neutrino masses are generated from the effective dimension-five operator $\kappa\left(\overline{\ell_{\mathrm{L}}} H\right)\left(H^{T} \ell_{\mathrm{L}}^{c}\right)$ [11], which can be obtained after integrating out the relevant heavy degrees of freedom from a full theory at a superhigh-energy scale. See, e.g., Z.Z. Xing and S. Zhou, Neutrinos in Particle Physics, Astronomy and Cosmology (Zhejiang University Press and Springer-Verlag, 2011).

[33] S. Antusch, J. Kersten, M. Lindner, and M. Ratz, Nucl. Phys. B 674, 401 (2003); S. Antusch et al., JHEP 0503, 024 (2005); J.W. Mei, Phys. Rev. D 71, 073012 (2005).

[34] L. Resnick, M.K. Sundaresan, and P.J.S. Watson, Phys. Rev. D 8, 172 (1973); J. Ellis, M.K. Gaillard, and D.V. Nanopoulos, Nucl. Phys. B 106, 292 (1976); J. Fleischer and F. Jegerlehner, Phys. Rev. D 23, 2001 (1981); D.Yu. Bardin, B.M. Vilenski, and P.Kh. Khristova, Sov. J. Nucl. Phys. 53, 152 (1991); A. Dabelstein and W. Hollik, Z. Phys. C 53, 507 (1992); B.A. Kniehl, Nucl. Phys. B 376, 3 (1992).

[35] E. Braaten and J.P. Leveille, Phys. Rev. D 22, 715 (1980); N. Sakai, Phys. Rev. D 22, 2220 (1980); T. Inami and T. Kubota, Nucl. Phys. B 179, 171 (1981); S.G. Gorishny, A.L. Kataev, and S.A. Larin, Sov. J. Nucl. Phys. 40, 329 (1984); M. Drees and K. Hikasa, Phys. Rev. D 41, 1547 (1990); Phys. Lett. B 240, 455 (1990); S.G. Gorishny et al., Mod. Phys. Lett. A 5, 2703 (1990); Phys. Rev. D 43, 1633 (1991); A.L. Kataev and V.T. Kim, Mod. Phys. Lett. A 9, 1309 (1994); L.R. Surguladze, Phys. Lett. 341, 61 (1994); K.G. Chetyrkin, Phys. Lett. B 390, 309 (1997); S.A. Larin, T. van Ritbergen, and J.A.M. Vermaseren, Phys. Lett. B 362, 134 (1995); K.G. Chetyrkin and A. Kwiatkowski, Nucl. Phys. B 461, 3 (1996).

[36] A. Djouadi, M. Spira, and P.M. Zerwas, Z. Phys. C 70, 427 (1996); M. Spira, Fortsch. Phys. 46, 203 (1998).

[37] A. Djouadi, J. Kalinowski, and M. Spira, Comput. Phys. Commun. 108, 56 (1998).

[38] A. Denner et al., Eur. Phys. J. C 71, 1753 (2011). 


\section{TABLES}

TABLE I. Running quark masses at some typical energy scales in the SM, including the Higgs mass $M_{H} \simeq 125 \mathrm{GeV}$ and the corresponding cutoff scale $\Lambda_{\mathrm{VS}} \simeq 4 \times 10^{12} \mathrm{GeV}$. Note that the values of the pole masses $M_{q}$ and running masses $m_{q}\left(M_{q}\right)$ themselves, rather than the running masses $m_{q}(\mu)$ at these mass scales, are given in the last two rows for comparison. But the pole masses of three light quarks are not listed, simply because the perturbative QCD calculation is not reliable in that energy region.

\begin{tabular}{c|c|c|c|c|c|c}
\hline \hline$\mu$ & $m_{u}(\mu)(\mathrm{MeV})$ & $m_{d}(\mu)(\mathrm{MeV})$ & $m_{s}(\mu)(\mathrm{MeV})$ & $m_{c}(\mu)(\mathrm{GeV})$ & $m_{b}(\mu)(\mathrm{GeV})$ & $m_{t}(\mu)(\mathrm{GeV})$ \\
\hline$m_{c}\left(m_{c}\right)$ & $2.79_{-0.82}^{+0.83}$ & $5.69_{-0.95}^{+0.96}$ & $116_{-24}^{+36}$ & $1.29_{-0.11}^{+0.05}$ & $5.95_{-0.15}^{+0.37}$ & $385.7_{-7.8}^{+8.1}$ \\
\hline $2 \mathrm{GeV}$ & $2.4_{-0.7}^{+0.7}$ & $4.9 \pm 0.8$ & $100_{-20}^{+30}$ & $1.11_{-0.14}^{+0.07}$ & $5.06_{-0.11}^{+0.29}$ & $322.2_{-4.9}^{+5.0}$ \\
\hline$m_{b}\left(m_{b}\right)$ & $2.02_{-0.60}^{+0.60}$ & $4.12_{-0.68}^{+0.69}$ & $84_{-17}^{+26}$ & $0.934_{-0.120}^{+0.058}$ & $4.19_{-0.16}^{+0.18}$ & $261.8_{-2.9}^{+3.0}$ \\
\hline$M_{W}$ & $1.39_{-0.41}^{+0.42}$ & $2.85_{-0.48}^{+0.49}$ & $58_{-12}^{+18}$ & $0.645_{-0.085}^{+0.043}$ & $2.90_{-0.06}^{+0.16}$ & $174.2 \pm 1.2$ \\
\hline$M_{Z}$ & $1.38_{-0.41}^{+0.42}$ & $2.82 \pm 0.48$ & $57_{-12}^{+18}$ & $0.638_{-0.084}^{+0.043}$ & $2.86_{-0.06}^{+0.16}$ & $172.1 \pm 1.2$ \\
\hline$M_{H}$ & $1.34_{-0.40}^{+0.40}$ & $2.74_{-0.47}^{+0.47}$ & $56_{-12}^{+17}$ & $0.621_{-0.082}^{+0.041}$ & $2.79_{-0.06}^{+0.15}$ & $167.0_{-1.2}^{+1.2}$ \\
\hline$m_{t}\left(m_{t}\right)$ & $1.31_{-0.39}^{+0.40}$ & $2.68 \pm 0.46$ & $55_{-11}^{+17}$ & $0.608_{-0.080}^{+0.041}$ & $2.73_{-0.06}^{+0.15}$ & $163.3 \pm 1.1$ \\
\hline $1 \mathrm{TeV}$ & $1.17 \pm 0.35$ & $2.40_{-0.41}^{+0.42}$ & $49_{-10}^{+15}$ & $0.543_{-0.072}^{+0.037}$ & $2.41_{-0.05}^{+0.14}$ & $148.1 \pm 1.3$ \\
\hline$\Lambda_{\mathrm{VS}}$ & $0.61_{-0.18}^{+0.19}$ & $1.27 \pm 0.22$ & $26_{-5}^{+8}$ & $0.281_{-0.04}^{+0.02}$ & $1.16_{-0.02}^{+0.07}$ & $82.6 \pm 1.4$ \\
\hline \hline$M_{q}$ & - & - & - & $1.84_{-0.13}^{+0.07}$ & $4.92_{-0.08}^{+0.21}$ & $172.9 \pm 1.1$ \\
\hline$m_{q}\left(M_{q}\right)$ & - & - & - & $1.14_{-0.12}^{+0.06}$ & $4.07_{-0.06}^{+0.18}$ & $162.5 \pm 1.1$ \\
\hline \hline
\end{tabular}

TABLE II. Running charged-lepton masses at some typical energy scales in the SM, including the Higgs mass $M_{H} \simeq 125 \mathrm{GeV}$ and the corresponding cutoff scale $\Lambda_{\mathrm{VS}} \simeq 4 \times 10^{12} \mathrm{GeV}$, where the uncertainties of $m_{l}(\mu)$ are determined by those of $M_{l}$. Note that the pole masses $M_{l}$, rather than the running masses $m_{l}\left(M_{l}\right)$, are given in the last row just for comparison.

\begin{tabular}{c|c|c|c}
\hline \hline$\mu$ & $m_{e}(\mu)(\mathrm{MeV})$ & $m_{\mu}(\mu)(\mathrm{MeV})$ & $m_{\tau}(\mu)(\mathrm{MeV})$ \\
\hline$m_{c}\left(m_{c}\right)$ & $0.495473903 \pm 0.000000013$ & $104.4617350_{-0.0000060}^{+0.000059}$ & $1774.62 \pm 0.16$ \\
\hline$m_{b}\left(m_{b}\right)$ & $0.493099926 \pm 0.000000013$ & $103.9961602_{-0.0000060}^{+0.000059}$ & $1767.02 \pm 0.16$ \\
\hline$M_{W}$ & $0.486845781_{-0.000000013}^{+0.00000013}$ & $102.7721083 \pm 0.00000059$ & $1747.05_{-0.16}^{+0.15}$ \\
\hline$M_{Z}$ & $0.486570154_{-0.000000012}^{+0.00000012}$ & $102.7181337_{-0.0000058}^{+0.000059}$ & $1746.17_{-0.16}^{+0.15}$ \\
\hline$M_{H}$ & $0.485858771_{-0.0000000013}^{+0.00000013}$ & $102.5788227_{-0.0000059}^{+0.000058}$ & $1743.89 \pm 0.16$ \\
\hline$m_{t}\left(m_{t}\right)$ & $0.485285152_{-0.0000000012}^{+0.00000012}$ & $102.4664851_{-0.0000058}^{+0.000059}$ & $1742.06 \pm 0.16$ \\
\hline $1 \mathrm{TeV}$ & $0.489535765_{-0.0000000013}^{+0.00000013}$ & $103.3441945 \pm 0.00000059$ & $1756.81 \pm 0.16$ \\
\hline$\Lambda_{\mathrm{VS}}$ & $0.484511554_{-0.000000012}^{+0.00000012}$ & $102.2835586_{-0.0000059}^{+0.0000058}$ & $1738.82 \pm 0.16$ \\
\hline \hline$M_{l}$ & $0.510998910 \pm 0.000000013$ & $105.658367 \pm 0.0000040$ & $1776.82 \pm 0.16$ \\
\hline \hline
\end{tabular}




\section{FIGURES}

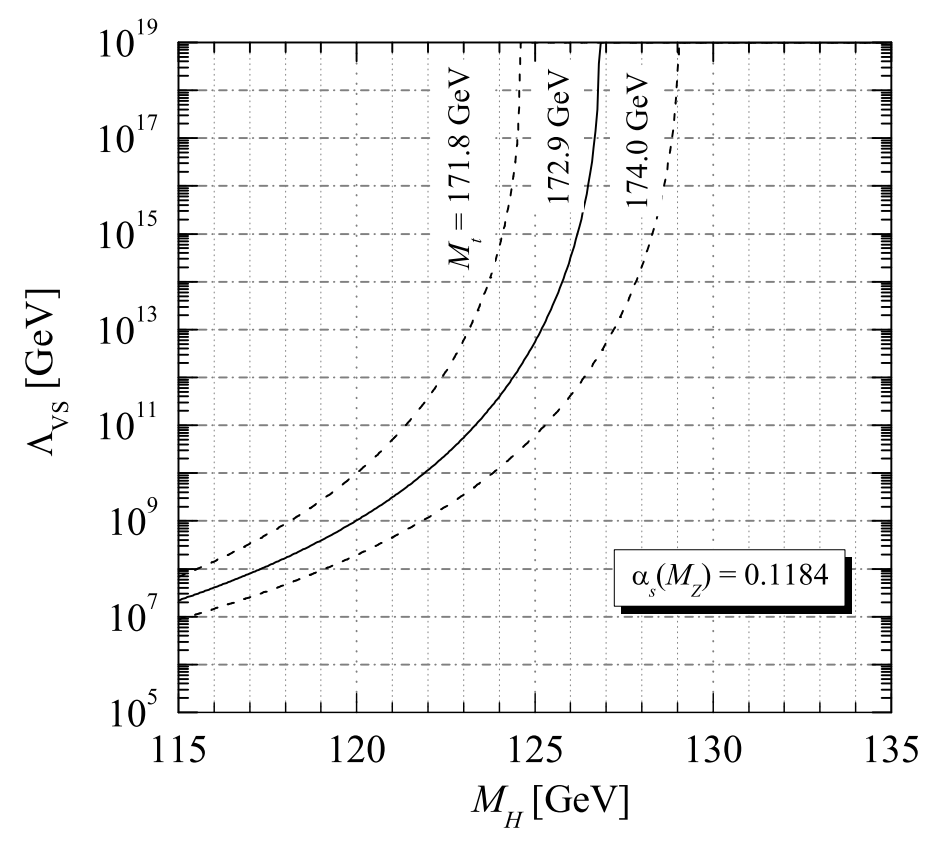

FIG. 1. Correlation between the energy scale $\Lambda_{\mathrm{VS}}$ and the Higgs mass $M_{H}$ based on the requirement of vacuum stability, where the solid curve corresponds to the best-fit value of the top-quark pole mass $M_{t}=172.9 \mathrm{GeV}$, and the dashed lines stand for the $1 \sigma$ lower and upper limits.

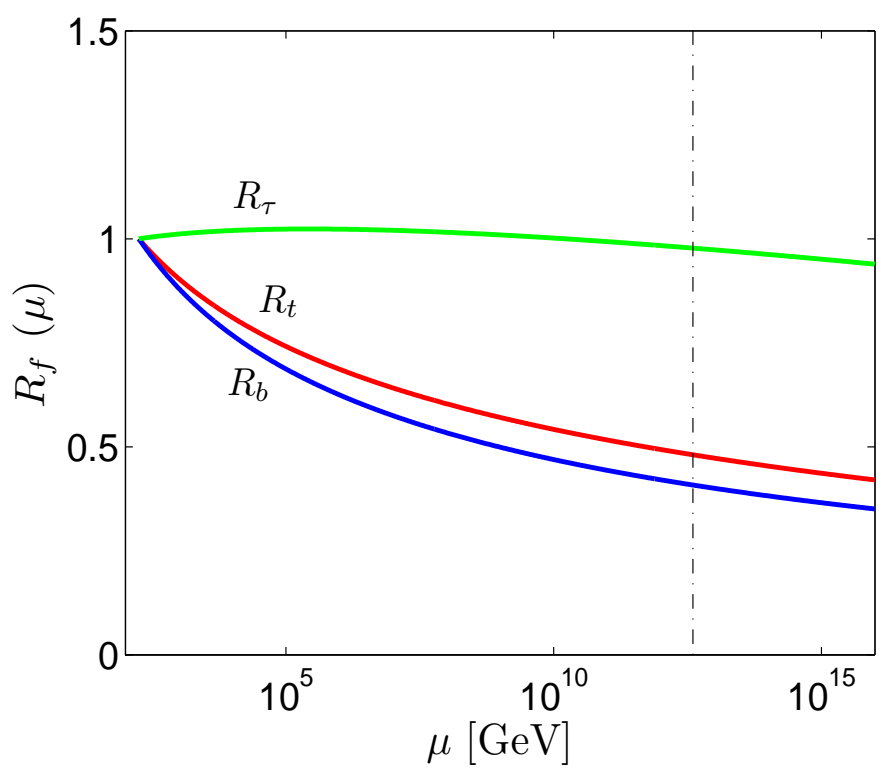

FIG. 2. The running behaviors of $R_{t}(\mu), R_{b}(\mu)$ and $R_{\tau}(\mu)$ with respect to the energy scale $\mu$ in the SM, where the vertical dashed line indicates the cutoff scale $\Lambda_{\mathrm{VS}} \simeq 4 \times 10^{12} \mathrm{GeV}$ as required by the vacuum stability for $M_{H} \simeq 125 \mathrm{GeV}$. 


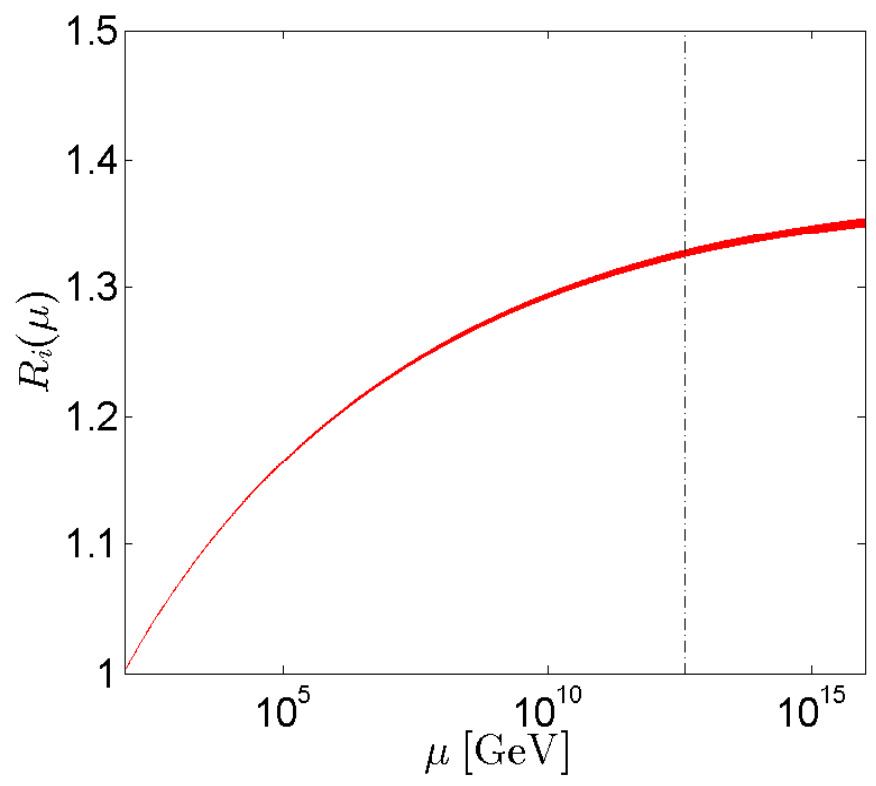

FIG. 3. The evolution of $R_{i}(\mu)$ with respect to the energy scale $\mu$, where the red band corresponds to the variation of the Higgs mass in the range $M_{H} \simeq(124 \cdots 126) \mathrm{GeV}$, and the vertical dashed line indicates the cutoff scale $\Lambda_{\mathrm{VS}} \simeq 4 \times 10^{12} \mathrm{GeV}$. Note that $R_{1}(\mu) \simeq R_{2}(\mu) \simeq R_{3}(\mu)$ holds to an excellent degree of accuracy.

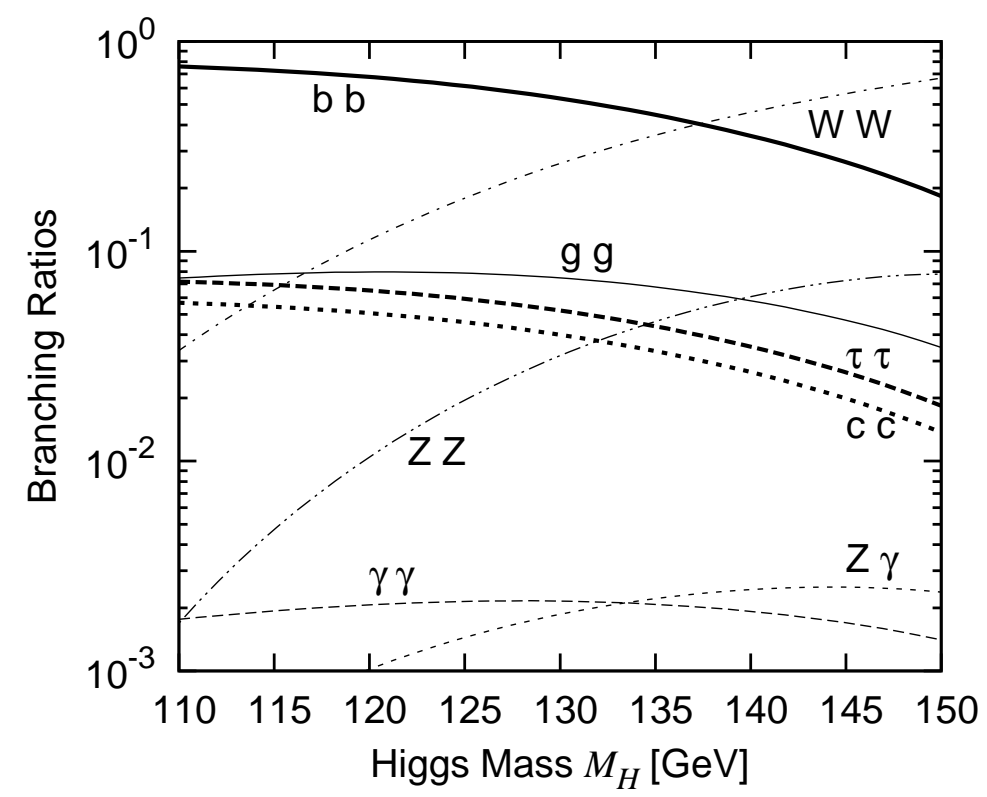

FIG. 4. The branching ratios of two-body Higgs decays versus the Higgs mass $M_{H}$. The thick lines stand for the dominant $H \rightarrow f \bar{f}$ modes: $b \bar{b}$ (solid line), $\tau^{+} \tau^{-}$(dashed line) and $c \bar{c}$ (dotted line); and the thin lines denote $H \rightarrow g g$ (solid line), $\gamma \gamma$ (dashed line), $Z \gamma$ (dotted line), $W^{+} W^{-}$ (dotted-dashed line) and $Z Z$ (double-dotted dashed line). 